\title{
A Computational Study of the Effect of Bending on Secondary Kinetic Isotope Effects in $\mathrm{S}_{\mathrm{N}} 2$ Transition States
}

\author{
Faraj Hasanayn, ${ }^{*}{ }^{\dagger}$ Andrew Streitwieser ${ }^{*},{ }^{\ddagger}$ and Rasha Al-Rifai ${ }^{\dagger}$
}

(a) Department of Chemistry, American University of Beirut, Beirut-Lebanon.

(b) Department of Chemistry, University of California, Berkeley, CA 94720-1460, USA.

Email: fh19@aub.edu.lb; astreit@socrates.bekeley.edu

\section{Supporting Information}

\section{Computation results at B3LYP 6-311++G**}

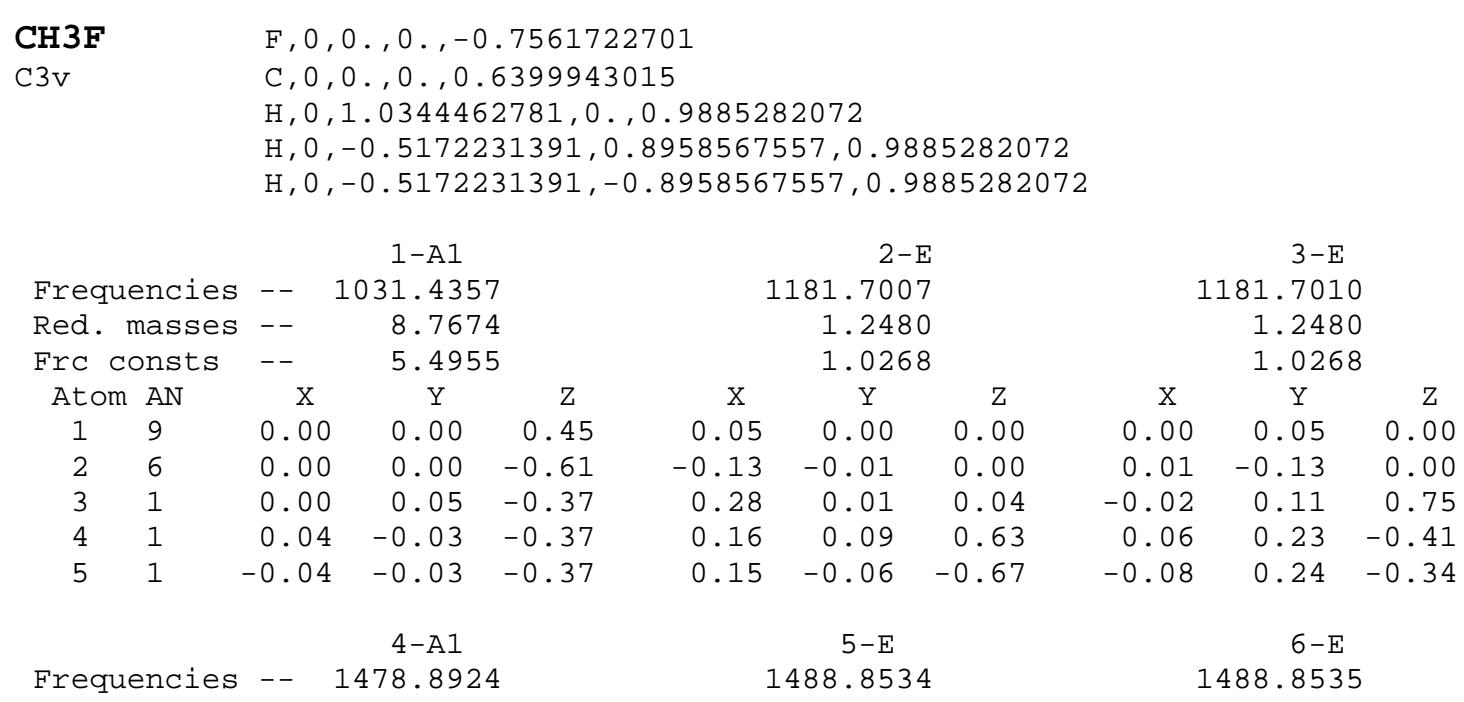

\footnotetext{
${ }^{\dagger}$ American University of Beirut.

${ }^{\ddagger}$ University of California, Berkeley.
} 


\begin{tabular}{|c|c|c|c|c|c|c|c|c|c|c|}
\hline \multirow{2}{*}{\multicolumn{2}{|c|}{$\begin{array}{l}\text { Red. masses } \\
\text { Frc consts }\end{array}$}} & -- & \multicolumn{2}{|c|}{1.1303} & \multicolumn{3}{|c|}{1.0508} & \multicolumn{3}{|c|}{1.0508} \\
\hline & & - & \multicolumn{2}{|c|}{1.4565} & \multicolumn{3}{|c|}{1.3724} & \multicolumn{3}{|c|}{1.3724} \\
\hline Atom & AN & $x$ & $\mathrm{Y}$ & Z & $X$ & $\mathrm{Y}$ & Z & $X$ & $\mathrm{Y}$ & Z \\
\hline 1 & 9 & 0.00 & 0.00 & 0.02 & 0.00 & 0.01 & 0.00 & -0.01 & 0.00 & 0.00 \\
\hline 2 & 6 & $\odot . \odot \odot$ & $\odot . \odot \odot$ & 0.10 & $\odot .0 \odot$ & $\odot .06$ & $\odot . \odot \odot$ & -0.06 & $\odot . \odot \odot$ & $\odot .0 \odot$ \\
\hline 3 & 1 & 0.00 & -0.20 & $-\odot .54$ & -0.03 & 0.14 & $\odot .33$ & 0.73 & 0.01 & 0.01 \\
\hline 4 & 1 & -0.17 & 0.10 & -0.54 & -0.38 & -0.53 & -0.15 & 0.06 & 0.36 & -0.29 \\
\hline 5 & 1 & 0.17 & 0.10 & -0.54 & 0.38 & -0.50 & -0.18 & 0.09 & -0.40 & 0.28 \\
\hline \multirow{2}{*}{\multicolumn{2}{|c|}{ Frequencies }} & \multicolumn{3}{|c|}{ 7-A1 } & \multicolumn{3}{|c|}{$8-E$} & \multicolumn{3}{|c|}{$9-E$} \\
\hline & & \multicolumn{3}{|c|}{3031.2205} & \multicolumn{3}{|c|}{3115.3256} & \multicolumn{3}{|c|}{3115.3281} \\
\hline \multicolumn{2}{|c|}{ Red. masses } & $5-$ & \multicolumn{2}{|c|}{1.0260} & \multicolumn{3}{|c|}{1.1084} & \multicolumn{3}{|c|}{1.1084} \\
\hline Frc & consts & - - & \multicolumn{2}{|c|}{5.5542} & \multicolumn{3}{|c|}{6.3379} & \multicolumn{3}{|c|}{6.3379} \\
\hline Atom & AN & $x$ & Y & Z & $x$ & $\mathrm{Y}$ & Z & $x$ & Y & Z \\
\hline 1 & 9 & 0.00 & 0.00 & 0.00 & $\odot .0 \odot$ & 0.00 & 0.00 & 0.00 & 0.00 & 0.00 \\
\hline 2 & 6 & 0.00 & $\odot .00$ & 0.04 & $\odot .0 \odot$ & 0.10 & $\odot .0 \odot$ & 0.10 & $\odot .0 \odot$ & $\odot .0 \odot$ \\
\hline 3 & 1 & 0.00 & $\odot .55$ & -0.17 & 0.00 & -0.77 & 0.24 & $\odot .02$ & 0.01 & 0.00 \\
\hline 4 & 1 & 0.48 & -0.27 & -0.17 & 0.34 & -0.17 & -0.12 & -0.58 & 0.35 & 0.21 \\
\hline 5 & 1 & -0.48 & -0.27 & -0.17 & -0.35 & -0.18 & -0.12 & -0.57 & -0.34 & -0.21 \\
\hline
\end{tabular}

Zero-point correction=

Sum of electronic and thermal Enthalpies=

Sum of electronic and thermal Free Energies=
$\odot .038987$ (Hartree/Particle) $-139.750008$
$-139.775305$

\begin{tabular}{|c|c|c|c|}
\hline & $\begin{array}{c}\text { E (Thermal) } \\
\text { KCAL/MOL }\end{array}$ & $\begin{array}{c}\text { CV } \\
\text { CAL/MOL-KELVIN }\end{array}$ & $\frac{\mathrm{S}}{\mathrm{CAL} / \mathrm{MOL}-\mathrm{KELVIN}}$ \\
\hline TOTAL & 26.295 & 6.977 & 53.242 \\
\hline ELECTRONIC & 0.000 & $\odot . \odot \odot \odot$ & 0.000 \\
\hline TRANSLATIONAL & $\odot .889$ & 2.981 & 36.504 \\
\hline ROTATIONAL & 0.889 & 2.981 & 16.529 \\
\hline VIBRATIONAL & 24.518 & 1.015 & 0.209 \\
\hline & $\mathrm{Q}$ & $\operatorname{LOG10}(\mathrm{Q})$ & LN(Q) \\
\hline TOTAL BOT & $\odot .845589 D-08$ & -8.072841 & -18.588403 \\
\hline TOTAL V $=0$ & ๑. 724120D+10 & 9.859811 & 22.703053 \\
\hline VIB (BOT) & ๑. 118648D-17 & -17.925739 & -41.275540 \\
\hline VIB $(V=0)$ & $\odot .101604 \mathrm{D}+01$ & ๑. 006912 & ๑. 015916 \\
\hline ELECTRONIC & $\odot .100000 \mathrm{D}+01$ & $\odot .00000 \odot$ & 0.000000 \\
\hline TRANSLATIONAL & $\odot .780006 \mathrm{D}+07$ & 6.892098 & 15.869642 \\
\hline ROTATIONAL & $\odot .913693 \mathrm{D}+03$ & 2.960800 & 6.817495 \\
\hline
\end{tabular}

CD3F

$\mathrm{F}, \odot, \odot ., 0,-\odot .7561722701$

C, $, 0,0 ., 0 ., 0.6399943015$

$\mathrm{D}, 0,1.0344462781,0 ., 0.9885282072$

$D, \odot,-\odot .5172231391, \odot .8958567557, \odot .9885282072$

D, $\odot,-\odot .5172231391,-\odot .8958567557, \odot .9885282072$

\begin{tabular}{|c|c|c|c|c|c|c|c|c|c|}
\hline Frequencies & \multicolumn{3}{|c|}{$1-E$} & \multicolumn{3}{|c|}{$2-E$} & \multicolumn{3}{|c|}{ 3-A1 } \\
\hline Red. masses & -- & \multicolumn{2}{|c|}{2.5402} & \multicolumn{3}{|c|}{2.5402} & \multicolumn{3}{|c|}{4.5911} \\
\hline Frc consts & -- & \multicolumn{2}{|c|}{1.2281} & \multicolumn{3}{|c|}{1.2281} & \multicolumn{3}{|c|}{2.6029} \\
\hline Atom AN & $\mathrm{X}$ & $\mathrm{Y}$ & Z & $x$ & $\mathrm{Y}$ & Z & $\mathrm{X}$ & $\mathrm{Y}$ & Z \\
\hline 19 & 0.10 & 0.00 & $\odot .00$ & $\odot .0 \odot$ & 0.10 & $\odot .0 \odot$ & $\odot .00$ & 0.00 & 0.33 \\
\hline 6 & -0.19 & -0.01 & 0.00 & 0.01 & -0.19 & 0.00 & 0.00 & 0.00 & -0.27 \\
\hline 3 & $\odot .09$ & $\odot . \odot \odot$ & 0.03 & $\odot .0 \odot$ & 0.05 & 0.79 & 0.00 & $-\odot . \odot 8$ & -0.52 \\
\hline 2 & 0.06 & 0.02 & 0.67 & 0.02 & 0.08 & -0.42 & -0.07 & 0.04 & -0.52 \\
\hline \multirow[t]{2}{*}{5} & 0.06 & -0.02 & -0.70 & $-\odot .02$ & 0.08 & $-\odot .37$ & 0.07 & 0.04 & -0.52 \\
\hline & \multicolumn{3}{|c|}{$4-E$} & \multicolumn{3}{|c|}{$5-E$} & \multicolumn{2}{|c|}{$6-\mathrm{A} 1$} & \\
\hline Frequencies & -- & \multicolumn{2}{|c|}{1078.9284} & \multicolumn{3}{|c|}{1078.9285} & \multicolumn{3}{|c|}{1135.7089} \\
\hline Red. masses & - & \multicolumn{2}{|c|}{2.2034} & \multicolumn{3}{|c|}{2.2034} & \multicolumn{3}{|c|}{3.3470} \\
\hline Frc consts & - & \multicolumn{2}{|c|}{1.5113} & \multicolumn{3}{|c|}{1.5113} & \multicolumn{3}{|c|}{2.5435} \\
\hline Atom AN & $x$ & $\mathrm{Y}$ & Z & X & $Y$ & Z & X & Y & Z \\
\hline 19 & $\odot .0 \odot$ & $\odot . \odot \odot$ & $0.0 \odot$ & $\odot .0 \odot$ & $\odot . \odot \odot$ & $\odot .0 \odot$ & $\odot . \odot \odot$ & $\odot . \odot \odot$ & -0.07 \\
\hline 6 & -0.14 & -0.02 & 0.00 & -0.02 & 0.14 & 0.00 & $\odot . \odot \odot$ & 0.00 & 0.35 \\
\hline
\end{tabular}




\begin{tabular}{|c|c|c|c|c|c|c|c|c|c|c|}
\hline 3 & 2 & 0.75 & -0.03 & $-\odot .03$ & 0.10 & 0.20 & 0.20 & $\odot .0 \odot$ & -0.27 & -0.47 \\
\hline 4 & 2 & 0.10 & 0.48 & -0.16 & -0.40 & -0.46 & -0.12 & -0.23 & 0.13 & -0.47 \\
\hline 5 & 2 & -0.02 & -0.34 & 0.19 & 0.42 & -0.57 & $-\odot .08$ & 0.23 & 0.13 & -0.47 \\
\hline \multicolumn{5}{|c|}{ 7-A1 } & \multicolumn{3}{|c|}{$8-E$} & \multicolumn{3}{|c|}{$9-E$} \\
\hline Red. & nasses & - & 2.0947 & & \multicolumn{3}{|c|}{2.3987} & \multicolumn{3}{|c|}{2.3987} \\
\hline \multicolumn{2}{|c|}{ Frc consts } & -- & 5.7953 & & \multicolumn{3}{|c|}{7.5679} & \multicolumn{3}{|c|}{7.5679} \\
\hline Atom & AN & $x$ & Y & Z & $X$ & Y & $Z$ & $x$ & Y & Z \\
\hline 1 & 9 & $\odot .0 \odot$ & 0.00 & $\odot .00$ & 0.00 & 0.00 & $\odot .00$ & 0.00 & $\odot .00$ & $\odot .0 \odot$ \\
\hline 2 & 6 & 0.00 & $\odot .0 \odot$ & $\odot .09$ & $\odot . \odot \odot$ & 0.20 & $\odot .00$ & 0.20 & $\odot . \odot \odot$ & 0.00 \\
\hline 3 & 2 & $\odot .0 \odot$ & 0.55 & -0.18 & 0.00 & $-\odot .77$ & 0.23 & $\odot . \odot \odot$ & 0.01 & $\odot .00$ \\
\hline 4 & 2 & 0.47 & -0.27 & -0.18 & 0.33 & -0.19 & -0.12 & -0.58 & 0.33 & 0.20 \\
\hline 5 & 2 & -0.47 & -0.27 & -0.18 & -0.34 & -0.19 & -0.12 & -0.57 & -0.33 & -0.20 \\
\hline
\end{tabular}

Zero-point correction=

Sum of electronic and thermal Enthalpies=

Sum of electronic and thermal Free Energies=

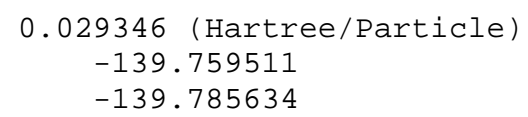

E (Thermal) KCAL/MOL

TOTAL

ELECTRONIC

TRANSLATIONAL

ROTATIONAL

VIBRATIONAL

TOTAL BOT

TOTAL $V=\odot$

VIB (BOT)

VIB $(\mathrm{V}=0)$

ELECTRONIC

TRANSLATIONAL

ROTATIONAL

$$
20.331
$$

0.000

$\odot .889$

0.889

18.554

Q

$\odot .476839 \mathrm{D}-03$

$\odot .150120 D+11$

$0.333753 D-13$

$\odot .105073 \mathrm{D}+01$

$\odot .100000 \mathrm{D}+01$

$\odot .886093 \mathrm{D}+07$

$\odot .161238 \mathrm{D}+04$
CV CAL/MOL - KELVIN 8.205

0.000

2. 981

2.981

2. 244

$\operatorname{LOG10}(\mathrm{Q})$

$-3.321628$

10.176438

$-13.476575$

0.021491

0.000000

6.947479

3. 207467
$S$

CAL/MOL-KELVIN

54.980

0.000

36.758

17.657

0.565

$\mathrm{LN}(\mathrm{Q})$

$-7.648331$

23.432114

$-31.030960$

0.049486

0.000000

15. 997162

7. 385466

\section{TS-inv-anion $\mathrm{F}-\mathrm{CH} 3-\mathrm{F}$}

D3h
$\mathrm{F}, \odot, \odot ., 0 .,-1.8623432313$

C, $0,0,0,0,0$.

$\mathrm{H}, 0,1.0727067059,0 ., 0$.

$\mathrm{H}, 0,-0.5363533529,0.9289912581, \odot$.

$\mathrm{H}, \odot,-\odot .5363533529,-\odot .9289912581, \odot$.

$\mathrm{F}, \odot, \odot ., \odot ., 1.8623432313$

$1-\mathrm{A} 2 "$

Frequencies - - $\quad-464.6162$

Red. masses - - 12.5501

Frc consts - - $\quad 1.5962$

Atom AN

$\begin{array}{lll}1 & 9 & 0.0 \odot \\ 2 & 6 & 0.0 \odot \\ 3 & 1 & 0.0 \odot \\ 4 & 1 & 0.0 \odot \\ 5 & 1 & 0.0 \odot \\ 6 & 9 & 0.0 \odot\end{array}$

$Y$

$0.0 \odot \quad-0.27$

$0.00 \quad 0.90$

$\odot . \odot \odot$

$\odot .0 \odot$

$-0.12$

$-0.12$

$4-\mathrm{A} 1^{\prime}$

Frequencies -

Red. masses - -

Frc consts --

Atom AN

$\begin{array}{rrr}1 & 9 & 0.0 \odot \\ 2 & 6 & 0.0 \odot \\ 3 & 1 & 0.0 \odot \\ 4 & 1 & -0.01 \\ 5 & 1 & 0.01\end{array}$

342.3406

18.9944

1.3116

Y. . $\odot \odot$

0.00

$-0.01$

$\odot .0 \odot$

$\odot . \odot \odot$

Z

0.71

0.00

$\odot .00$

0.00

$\odot .0 \odot$
2-E'

313.6657

4.5366

0.2630

$\mathrm{X}$
$\odot .0 \odot$

$\mathrm{Y}$

0.00

$\odot .0 \odot$

$-0.01$

0.01

$\odot .0 \odot$
$Z$
$\odot . \odot \odot$
$\odot . \odot \odot$
$\odot . \odot \odot$
$\odot . \odot \odot$
$\odot . \odot \odot$
$\odot . \odot \odot$

$$
5-E^{\prime \prime}
$$

1043.4603

1. 0204

0.6546

$Y$

0.00

$\odot .00$

$\odot .0 \odot$

$\odot .0 \odot$

$\odot .00$

\subsection{2}

0.00

$\odot . \odot \odot$

$0.00 \quad-0.41$

$0.00 \quad-0.41$

\begin{tabular}{ccc} 
& \multicolumn{3}{c}{$3-E^{\prime}$} \\
& 313.6688 & \\
& 4.5367 & \\
& 0.2630 & \\
$X$ & $Y$ & $Z$ \\
0.18 & $0.0 \odot$ & $0.0 \odot$ \\
-0.46 & $0.0 \odot$ & $0.0 \odot$ \\
$-0.5 \odot$ & $0.0 \odot$ & $0.0 \odot$ \\
-0.48 & -0.01 & $\odot . \odot \odot$ \\
-0.48 & 0.01 & $0.0 \odot$ \\
0.18 & $0.0 \odot$ & $\odot . \odot \odot$
\end{tabular}

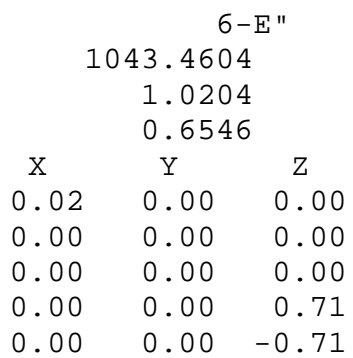




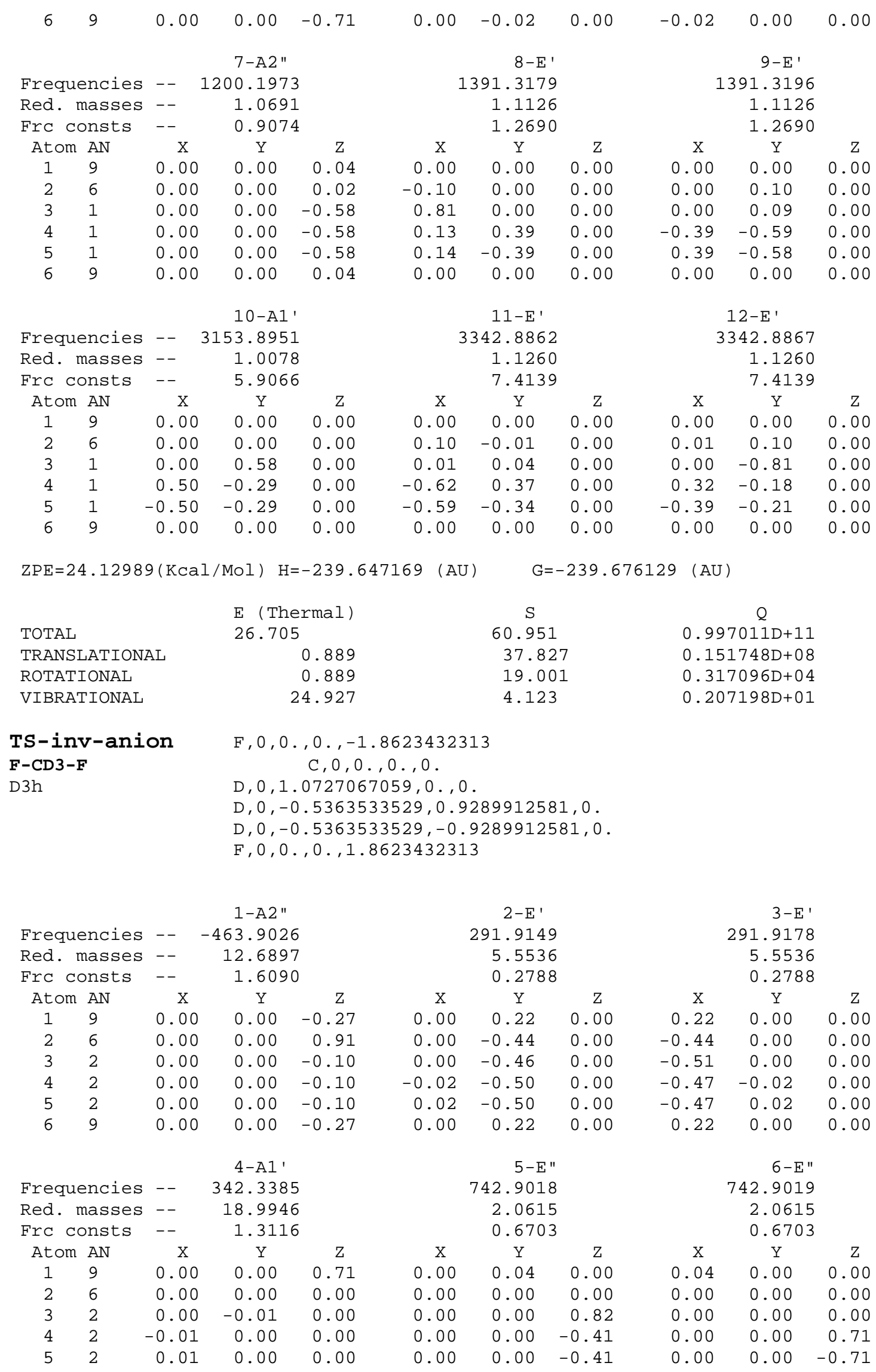




\begin{tabular}{|c|c|c|c|c|c|c|c|c|c|c|}
\hline \multirow[t]{2}{*}{6} & 9 & 0.00 & 0.00 & $-\odot .71$ & 0.00 & -0.04 & 0.00 & -0.04 & $\odot .0 \odot$ & $\odot .0 \odot$ \\
\hline & & \multicolumn{3}{|c|}{$7-A 2^{\prime \prime}$} & \multicolumn{3}{|c|}{$8-E^{\prime}$} & \multicolumn{3}{|c|}{$9-E^{\prime}$} \\
\hline \multicolumn{2}{|c|}{ Frequencies } & - & \multicolumn{2}{|l|}{874.1718} & \multicolumn{3}{|c|}{1028.6185} & \multicolumn{3}{|c|}{1028.6203} \\
\hline \multicolumn{2}{|c|}{ Red. masses } & - & \multicolumn{2}{|l|}{2.2392} & \multicolumn{3}{|c|}{2.3047} & \multicolumn{3}{|c|}{2.3047} \\
\hline \multicolumn{2}{|c|}{ Frc consts } & - - & \multicolumn{2}{|c|}{1.0082} & \multicolumn{3}{|c|}{1.4367} & \multicolumn{3}{|c|}{1.4368} \\
\hline Atom & AN & $x$ & Y & Z & $x$ & $\mathrm{Y}$ & Z & $x$ & Y & Z \\
\hline 1 & 9 & $\odot . \odot \odot$ & $\odot .0 \odot$ & 0.08 & $\odot .01$ & $\odot . \odot \odot$ & $\odot . \odot \odot$ & $\odot .0 \odot$ & $-\odot . \odot 1$ & $\odot .0 \odot$ \\
\hline 2 & 6 & $\odot . \odot \odot$ & $\odot . \odot \odot$ & $\odot . \odot 4$ & -0.17 & $\odot . \odot \odot$ & $\odot . \odot \odot$ & $\odot . \odot \odot$ & $\odot .17$ & $\odot . \odot \odot$ \\
\hline 3 & 2 & $0.0 \odot$ & 0.00 & $-\odot .57$ & 0.78 & 0.00 & 0.00 & $\odot .0 \odot$ & 0.18 & 0.00 \\
\hline 4 & 2 & $\odot .0 \odot$ & 0.00 & $-\odot .57$ & 0.06 & 0.42 & $\odot .0 \odot$ & -0.42 & -0.54 & $\odot .0 \odot$ \\
\hline 5 & 2 & $\odot . \odot \odot$ & $\odot .0 \odot$ & $-\odot .57$ & $\odot .06$ & -0.42 & $\odot . \odot \odot$ & 0.42 & -0.54 & $\odot .0 \odot$ \\
\hline \multirow[t]{2}{*}{6} & 9 & 0.00 & 0.00 & $\odot .08$ & 0.01 & 0.00 & 0.00 & 0.00 & $-\odot .01$ & 0.00 \\
\hline & & \multicolumn{3}{|c|}{$10-\mathrm{A} 1^{\prime}$} & \multicolumn{3}{|c|}{$11-E^{\prime}$} & \multicolumn{3}{|c|}{$12-E^{\prime}$} \\
\hline \multicolumn{2}{|c|}{ Frequencies } & - & \multicolumn{2}{|l|}{2231.0108} & \multicolumn{3}{|c|}{2499.3809} & \multicolumn{3}{|c|}{2499.3816} \\
\hline \multicolumn{2}{|c|}{ Red. masses } & - & \multicolumn{2}{|l|}{2.0141} & \multicolumn{3}{|c|}{2.4513} & \multicolumn{3}{|c|}{2.4513} \\
\hline \multicolumn{2}{|c|}{ Frc consts } & - - & \multicolumn{2}{|c|}{5.9067} & \multicolumn{3}{|c|}{9.0222} & \multicolumn{3}{|c|}{9.0222} \\
\hline Atom & AN & $x$ & Y & Z & $x$ & $\mathrm{Y}$ & Z & $x$ & $\mathrm{Y}$ & Z \\
\hline 1 & 9 & $\odot .0 \odot$ & 0.00 & 0.00 & $\odot .00$ & 0.00 & 0.00 & $\odot .00$ & 0.00 & 0.00 \\
\hline 2 & 6 & $\odot .0 \odot$ & $\odot .0 \odot$ & $\odot .0 \odot$ & 0.21 & -0.01 & $\odot .0 \odot$ & 0.01 & 0.21 & $\odot .0 \odot$ \\
\hline 3 & 2 & $\odot . \odot \odot$ & $\odot .58$ & $\odot . \odot \odot$ & $-\odot .01$ & 0.04 & $\odot .0 \odot$ & $\odot . \odot \odot$ & $-\odot .8 \odot$ & $\odot . \odot \odot$ \\
\hline 4 & 2 & 0.50 & -0.29 & $\odot .00$ & -0.62 & 0.35 & 0.00 & 0.31 & -0.19 & 0.00 \\
\hline 5 & 2 & -0.50 & -0.29 & 0.00 & -0.58 & -0.33 & 0.00 & -0.37 & -0.22 & 0.00 \\
\hline 6 & 9 & $\odot .0 \odot$ & $\odot . \odot \odot$ & $\odot . \odot \odot$ & $\odot .0 \odot$ & $\odot .0 \odot$ & $\odot .0 \odot$ & $\odot . \odot \odot$ & $\odot .0 \odot$ & $\odot .0 \odot$ \\
\hline
\end{tabular}

$\mathrm{ZPE}=17.97424(\mathrm{Kcal} / \mathrm{Mol}) \mathrm{H}=-239.656700(\mathrm{AU}) \quad \mathrm{G}=--239.686479(\mathrm{AU})$

$\begin{array}{lccc} & \text { E }(\text { Thermal }) & \mathrm{S} & \mathrm{Q} \\ \text { TOTAL } & 20.724 & 62.674 & 0.176695 \mathrm{D}+12 \\ \text { TRANSLATIONAL } & 0.889 & 37.992 & 0.164891 \mathrm{D}+08 \\ \text { ROTATIONAL } & \odot .889 & 19.715 & 0.454100 \mathrm{D}+04 \\ \text { VIBRATIONAL } & 18.947 & 4.967 & 0.235980 \mathrm{D}+01\end{array}$

\section{TS - ret - anion F-CH3-F Cs}

$C, \odot,-\odot .0595877015, \odot ., 0.912839425$

$\mathrm{H}, 0,0.9967272979,0 ., 1.1417969153$

$\mathrm{H}, \odot,-\odot .5675787081,0.8910317663,1.2832565312$

$\mathrm{H}, \odot,-\odot .5675787081,-0.8910317663,1.2832565312$

$\mathrm{F}, \odot, \odot .0275531293,1.2217744973,-\odot .5102970293$

F, $\odot, 0.0275531293,-1.2217744973,-\odot .5102970293$

\begin{tabular}{|c|c|c|c|c|c|c|c|c|c|}
\hline \multirow{2}{*}{ Frequencies } & \multicolumn{3}{|c|}{$1-A^{\prime \prime}$} & \multicolumn{3}{|c|}{$2-A^{\prime \prime}$} & \multicolumn{3}{|c|}{$3-A^{\prime}$} \\
\hline & -- & \multicolumn{2}{|c|}{-630.4037} & \multicolumn{3}{|c|}{188.1776} & \multicolumn{3}{|c|}{269.6231} \\
\hline Red. masses & - & \multicolumn{2}{|c|}{3.0206} & \multicolumn{3}{|c|}{ 1. 2101} & \multicolumn{3}{|c|}{17.0439} \\
\hline Frc consts & -- & \multicolumn{2}{|c|}{0.7073} & \multicolumn{3}{|c|}{$\odot .0252$} & \multicolumn{3}{|c|}{$\odot .730 \odot$} \\
\hline Atom AN & $\mathrm{X}$ & $\mathrm{Y}$ & Z & X & $\mathrm{Y}$ & Z & X & $\mathrm{Y}$ & Z \\
\hline 19 & $\odot .0 \odot$ & $\odot .0 \odot$ & 0.31 & $\odot .00$ & $\odot .00$ & $\odot .08$ & $\odot .00$ & 0.12 & $\odot .0 \odot$ \\
\hline 1 & $\odot .0 \odot$ & $\odot . \odot \odot$ & -0.14 & $\odot .0 \odot$ & $\odot .0 \odot$ & 0.65 & $-\odot .03$ & -0.20 & $\odot . \odot \odot$ \\
\hline 1 & -0.32 & 0.47 & 0.30 & 0.50 & -0.03 & -0.18 & 0.01 & -0.16 & 0.06 \\
\hline 1 & 0.32 & -0.47 & 0.30 & -0.50 & 0.03 & -0.18 & 0.01 & -0.16 & -0.06 \\
\hline 9 & 0.02 & 0.12 & -0.11 & -0.04 & 0.04 & -0.03 & 0.00 & $-\odot . \odot 2$ & 0.66 \\
\hline \multirow[t]{2}{*}{6} & $-\odot . \odot 2$ & -0.12 & -0.11 & $\odot . \odot 4$ & -0.04 & -0.03 & 0.00 & -0.02 & -0.66 \\
\hline & \multicolumn{3}{|c|}{$4-A^{\prime}$} & \multicolumn{3}{|c|}{$5-A^{\prime}$} & \multicolumn{3}{|c|}{$6-A^{\prime \prime}$} \\
\hline Frequencies & -- & \multicolumn{2}{|c|}{532.5965} & \multicolumn{3}{|c|}{905.2558} & \multicolumn{3}{|c|}{1032.7191} \\
\hline Red. masses & -- & \multicolumn{2}{|c|}{8.2688} & \multicolumn{3}{|c|}{1.1860} & \multicolumn{3}{|c|}{1.1350} \\
\hline Frc consts & -- & \multicolumn{2}{|c|}{1.3819} & \multicolumn{3}{|c|}{0.5726} & \multicolumn{3}{|c|}{0.7132} \\
\hline Atom AN & $X$ & $\mathrm{Y}$ & Z & $\mathrm{X}$ & Y & Z & $\mathrm{X}$ & Y & Z \\
\hline 16 & -0.03 & 0.68 & $0.0 \odot$ & -0.11 & -0.05 & $\odot .0 \odot$ & $0.0 \odot$ & 0.00 & -0.01 \\
\hline 1 & -0.04 & 0.58 & 0.00 & 0.01 & 0.86 & $\odot .0 \odot$ & 0.00 & $\odot .0 \odot$ & -0.07 \\
\hline 1 & 0.05 & 0.15 & 0.12 & 0.16 & -0.31 & $-\odot .04$ & 0.07 & 0.63 & -0.31 \\
\hline 1 & 0.05 & 0.15 & -0.12 & 0.16 & -0.31 & 0.04 & -0.07 & -0.63 & -0.31 \\
\hline 9 & 0.01 & -0.24 & -0.04 & 0.03 & 0.01 & 0.01 & -0.01 & -0.05 & 0.02 \\
\hline 6 & 0.01 & -0.24 & 0.04 & 0.03 & 0.01 & -0.01 & $\odot .01$ & $\odot .05$ & 0.02 \\
\hline
\end{tabular}




\begin{tabular}{|c|c|c|c|c|c|c|c|c|c|c|}
\hline \multirow{2}{*}{\multicolumn{2}{|c|}{ Frequencies }} & \multicolumn{3}{|c|}{$7-A^{\prime}$} & \multicolumn{3}{|c|}{$8-A^{\prime \prime}$} & \multicolumn{3}{|c|}{$9-A^{\prime}$} \\
\hline & & - - & \multicolumn{2}{|c|}{$\begin{array}{c}1268.2047 \\
1268\end{array}$} & \multicolumn{3}{|c|}{1424.7193} & \multicolumn{3}{|c|}{1505.6329} \\
\hline Red. & nasses & -- & \multicolumn{2}{|c|}{1.1003} & \multicolumn{3}{|c|}{1.0890} & \multicolumn{3}{|c|}{1.0764} \\
\hline \multicolumn{2}{|c|}{ Frc consts } & -- & \multicolumn{2}{|c|}{1.0426} & \multicolumn{3}{|c|}{1.3024} & \multicolumn{3}{|c|}{1.4377} \\
\hline Atom & AN & $x$ & $\mathrm{Y}$ & Z & $x$ & $\mathrm{Y}$ & Z & $X$ & Y & Z \\
\hline 1 & 6 & 0.04 & -0.07 & 0.00 & 0.00 & 0.00 & $-\odot .09$ & $\odot .08$ & 0.00 & 0.00 \\
\hline 2 & 1 & 0.11 & 0.49 & $\odot .0 \odot$ & $\odot .00$ & 0.00 & 0.79 & 0.08 & 0.17 & 0.00 \\
\hline 3 & 1 & -0.23 & 0.56 & $-\odot .09$ & -0.39 & 0.13 & 0.10 & -0.51 & -0.25 & 0.39 \\
\hline 4 & 1 & -0.23 & 0.56 & $\odot .09$ & 0.39 & -0.13 & 0.10 & -0.51 & -0.25 & -0.39 \\
\hline 5 & 9 & $0.0 \odot$ & -0.02 & 0.02 & 0.00 & $0.0 \odot$ & $\odot .00$ & $\odot .0 \odot$ & 0.01 & 0.00 \\
\hline \multirow[t]{2}{*}{6} & 9 & 0.00 & -0.02 & -0.02 & 0.00 & 0.00 & $\odot . \odot \odot$ & 0.00 & 0.01 & $\odot .0 \odot$ \\
\hline & & \multicolumn{3}{|c|}{$10-A^{\prime}$} & \multicolumn{3}{|c|}{$11-A^{\prime \prime}$} & \multicolumn{3}{|c|}{$12-A^{\prime}$} \\
\hline \multicolumn{2}{|c|}{ Frequencies } & -- & \multicolumn{2}{|c|}{3010.5323} & \multicolumn{3}{|c|}{3053.4129} & \multicolumn{3}{|c|}{3210.4745} \\
\hline \multicolumn{2}{|c|}{ Red. masses } & -- & \multicolumn{2}{|c|}{ 1. 0281} & \multicolumn{3}{|c|}{1.1100} & \multicolumn{3}{|c|}{1.1043} \\
\hline \multicolumn{2}{|c|}{ Frc consts } & -- & \multicolumn{2}{|c|}{5.4898} & \multicolumn{3}{|c|}{6.0976} & \multicolumn{3}{|c|}{6.7065} \\
\hline Atom & AN & $\mathrm{X}$ & $\mathrm{Y}$ & Z & $X$ & $\mathrm{Y}$ & Z & $x$ & Y & Z \\
\hline 1 & 6 & -0.02 & -0.03 & 0.00 & 0.00 & 0.00 & -0.10 & $-\odot .09$ & 0.00 & 0.00 \\
\hline 2 & 1 & -0.30 & 0.04 & 0.00 & 0.00 & 0.00 & -0.03 & 0.95 & -0.12 & 0.00 \\
\hline 3 & 1 & 0.29 & 0.20 & 0.57 & 0.33 & 0.23 & 0.58 & 0.07 & $\odot .06$ & 0.17 \\
\hline 4 & 1 & 0.29 & 0.20 & -0.57 & -0.33 & -0.23 & 0.58 & 0.07 & 0.06 & -0.17 \\
\hline 5 & 9 & $0.0 \odot$ & $\odot .00$ & $\odot .0 \odot$ & 0.00 & 0.00 & $\odot . \odot \odot$ & $\odot .00$ & $\odot .0 \odot$ & $\odot .0 \odot$ \\
\hline 6 & 9 & $\odot .0 \odot$ & $\odot .00$ & 0.00 & 0.00 & $\odot .00$ & 0.00 & $\odot .0 \odot$ & 0.00 & $\odot .00$ \\
\hline
\end{tabular}

$\mathrm{ZPE}=23.44691(\mathrm{Kcal} / \mathrm{Mol}) \mathrm{H}=-239.576296(\mathrm{AU}) \quad \mathrm{G}-239.607564(\mathrm{AU})$

$\begin{array}{lrcc} & \mathrm{E}(\mathrm{Thermal)} & \mathrm{S} & \mathrm{Q} \\ \text { TOTAL } & 26.071 & 65.810 & 0.105797 \mathrm{D}+13 \\ \text { TRANSLATIONAL } & 0.889 & 37.827 & 0.151748 \mathrm{D}+08 \\ \text { ROTATIONAL } & 0.889 & 23.280 & 0.273082 \mathrm{D}+05 \\ \text { VIBRATIONAL } & 24.294 & 4.703 & 0.255303 \mathrm{D}+01\end{array}$

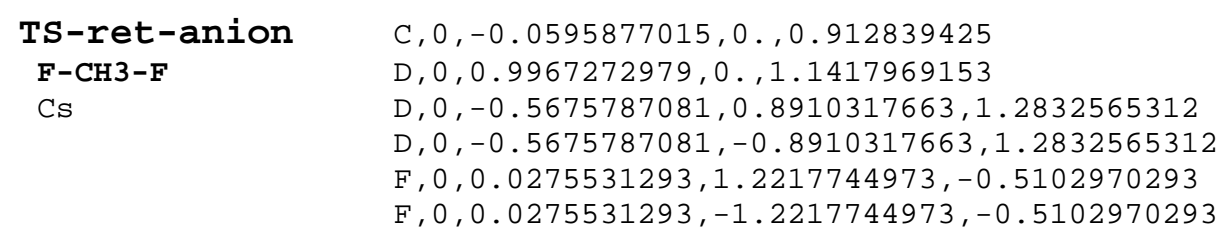

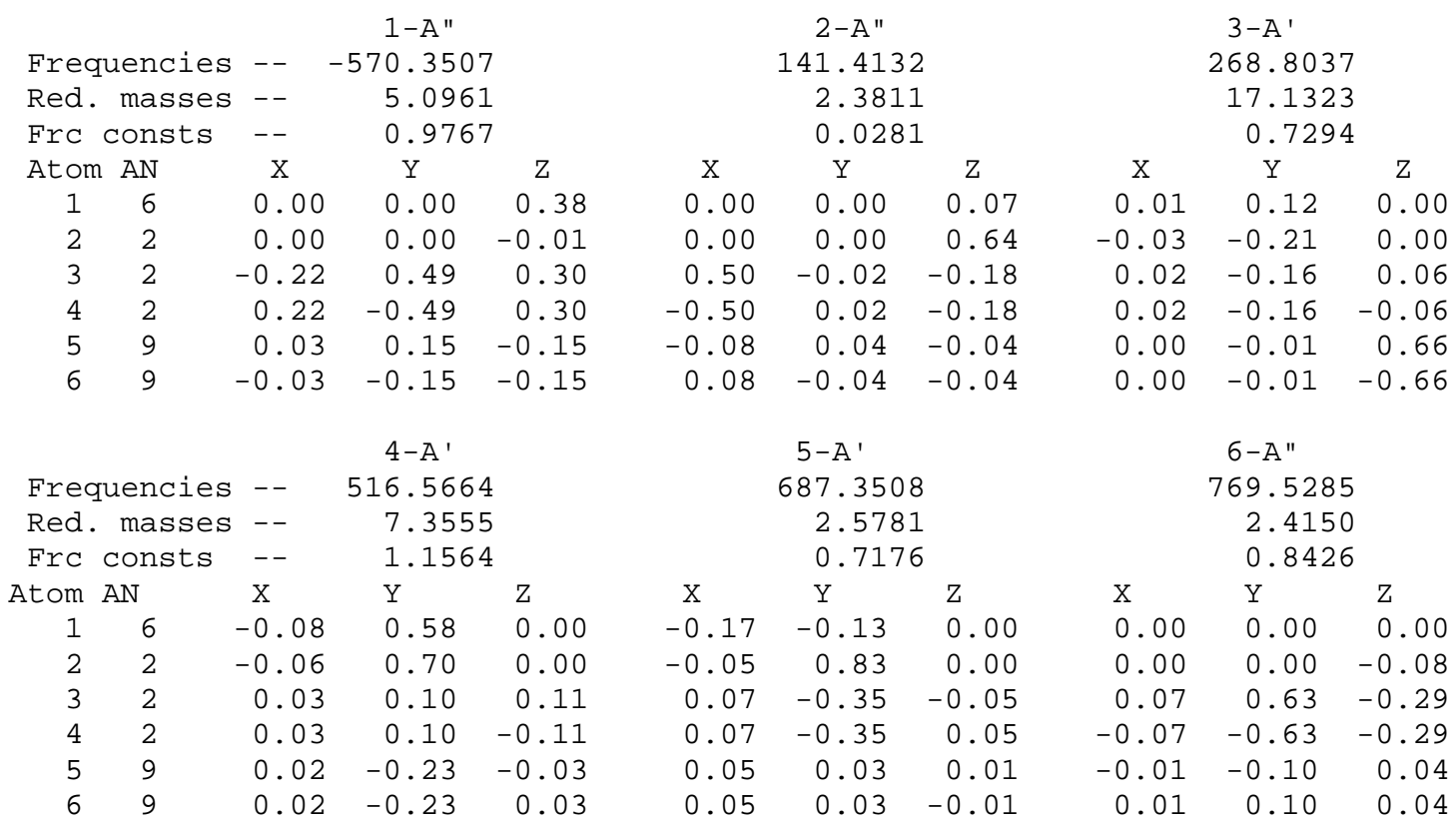




\begin{tabular}{|c|c|c|c|c|c|c|c|c|c|}
\hline \multirow{2}{*}{ Frequencies } & \multicolumn{3}{|c|}{$7-A^{\prime}$} & \multicolumn{3}{|c|}{$8-A^{\prime \prime}$} & \multicolumn{3}{|c|}{$9-A^{\prime}$} \\
\hline & - & \multicolumn{2}{|c|}{937.2094} & \multicolumn{3}{|c|}{1041.6712} & \multicolumn{3}{|c|}{1099.5574} \\
\hline Red. masses & - & \multicolumn{2}{|c|}{2.3518} & \multicolumn{3}{|c|}{2.2127} & \multicolumn{3}{|c|}{2.2305} \\
\hline Frc consts & -- & \multicolumn{2}{|c|}{1.2171} & \multicolumn{3}{|c|}{1.4146} & \multicolumn{3}{|c|}{1.5889} \\
\hline Atom AN & $x$ & $\mathrm{Y}$ & Z & $\mathrm{X}$ & Y & Z & $x$ & Y & Z \\
\hline 16 & $\odot .06$ & -0.15 & $\odot . \odot \odot$ & $\odot . \odot \odot$ & $\odot . \odot \odot$ & $-0 . \overline{14}$ & 0.14 & $\odot . \odot \odot$ & $\odot . \odot \odot$ \\
\hline 2 & 0.14 & 0.46 & $\odot . \odot \odot$ & 0.00 & 0.00 & 0.77 & 0.17 & 0.15 & 0.00 \\
\hline 2 & -0.19 & 0.56 & -0.12 & -0.42 & 0.11 & 0.05 & -0.51 & -0.20 & 0.41 \\
\hline 2 & -0.19 & 0.56 & 0.12 & 0.42 & -0.11 & 0.05 & -0.51 & -0.20 & -0.41 \\
\hline 9 & -0.01 & -0.04 & 0.03 & 0.00 & 0.00 & 0.00 & $\odot . \odot \odot$ & 0.01 & -0.01 \\
\hline \multirow[t]{2}{*}{6} & -0.01 & -0.04 & -0.03 & 0.00 & 0.00 & 0.00 & 0.00 & 0.01 & 0.01 \\
\hline & \multicolumn{3}{|c|}{$10-A^{\prime}$} & \multicolumn{3}{|c|}{$11-A^{\prime \prime}$} & \multicolumn{3}{|c|}{$12-A^{\prime}$} \\
\hline Frequencies & - - & \multicolumn{2}{|c|}{2149.3619} & \multicolumn{3}{|c|}{2266.3489} & \multicolumn{3}{|c|}{2383.1443} \\
\hline Red. masses & - - & \multicolumn{2}{|c|}{2.0722} & \multicolumn{3}{|c|}{2.3795} & \multicolumn{3}{|c|}{2.3974} \\
\hline Frc consts & - & \multicolumn{2}{|c|}{5.6404} & \multicolumn{3}{|c|}{7.2008} & \multicolumn{3}{|c|}{8.0221} \\
\hline Atom AN & $x$ & $\mathrm{Y}$ & Z & X & Y & Z & $\mathrm{x}$ & Y & Z \\
\hline 16 & -0.03 & -0.07 & 0.00 & 0.00 & 0.00 & 0.19 & -0.20 & -0.01 & 0.00 \\
\hline 2 & -0.41 & 0.05 & 0.00 & 0.00 & 0.00 & 0.01 & 0.90 & -0.11 & $\odot .0 \odot$ \\
\hline 2 & 0.28 & 0.20 & 0.54 & -0.31 & -0.23 & -0.57 & 0.12 & 0.08 & 0.23 \\
\hline 2 & 0.28 & 0.20 & -0.54 & 0.31 & 0.23 & -0.57 & 0.12 & 0.08 & -0.23 \\
\hline 5 & $0.0 \odot$ & $\odot . \odot \odot$ & $\odot .0 \odot$ & $0.0 \odot$ & $\odot . \odot \odot$ & 0.00 & $\odot . \odot \odot$ & $\odot .0 \odot$ & $0.0 \odot$ \\
\hline 6 & $\odot .0 \odot$ & $\odot .00$ & $\odot .0 \odot$ & $\odot .0 \odot$ & $\odot .0 \odot$ & 0.00 & $\odot .0 \odot$ & 0.00 & $\odot .0 \odot$ \\
\hline
\end{tabular}

$\begin{array}{lccc}\text { ZPE= } 17.52792 & (\mathrm{Kcal} / \mathrm{Mol}) \mathrm{H}=-239.585438(\mathrm{AU}) & \mathrm{G}=-239.617487(\mathrm{AU}) \\ & \mathrm{E}(\mathrm{Thermal}) & \mathrm{S} & \mathrm{Q} \\ \text { TOTAL } & 20.335 & 67.454 & 0.177831 \mathrm{D}+13 \\ \text { TRANSLATIONAL } & 0.889 & 37.992 & 0.164891 \mathrm{D}+08 \\ \text { ROTATIONAL } & 0.889 & 23.637 & 0.326877 \mathrm{D}+05 \\ \text { VIBRATIONAL } & 18.557 & 5.825 & 0.329933 \mathrm{D}+01\end{array}$

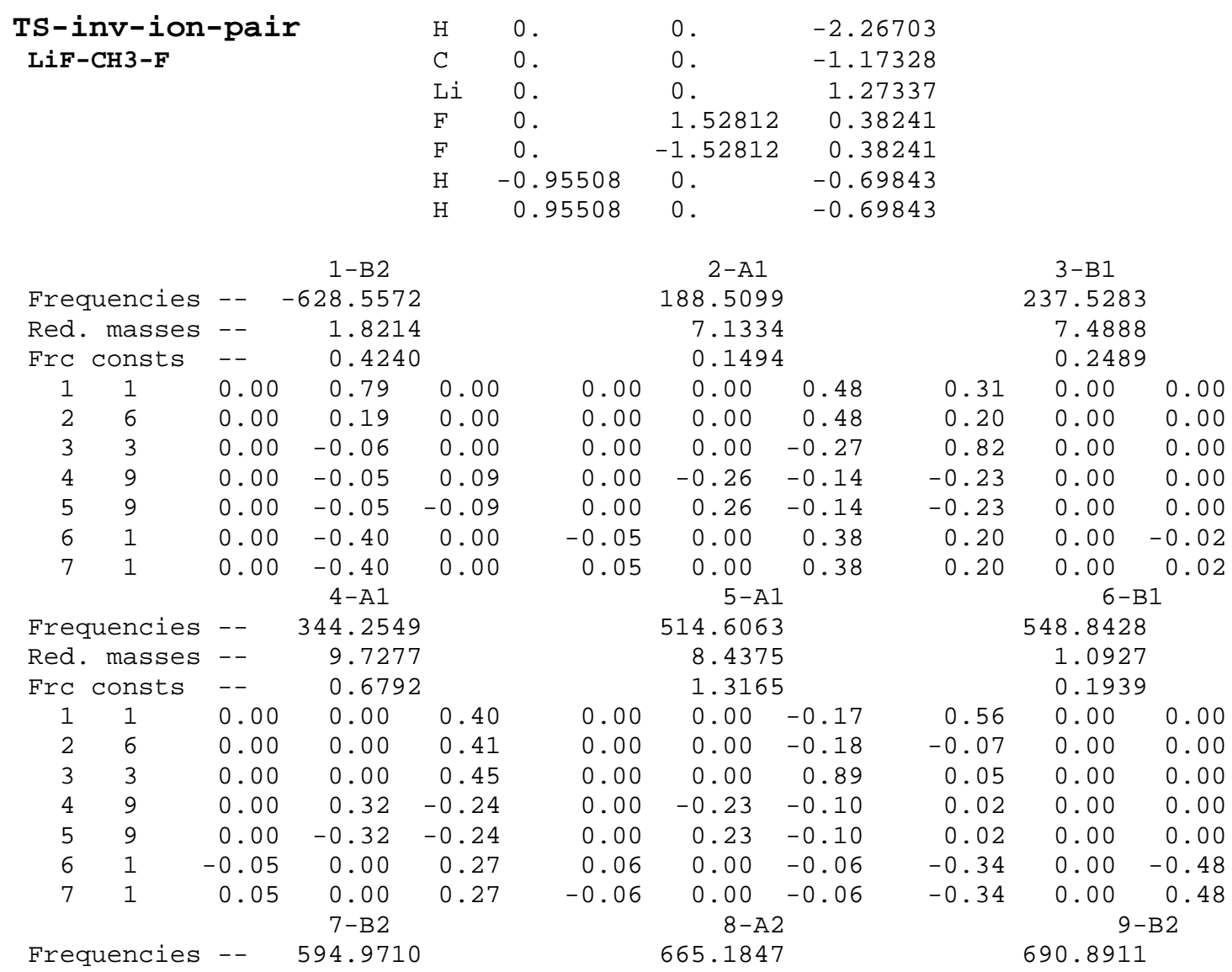




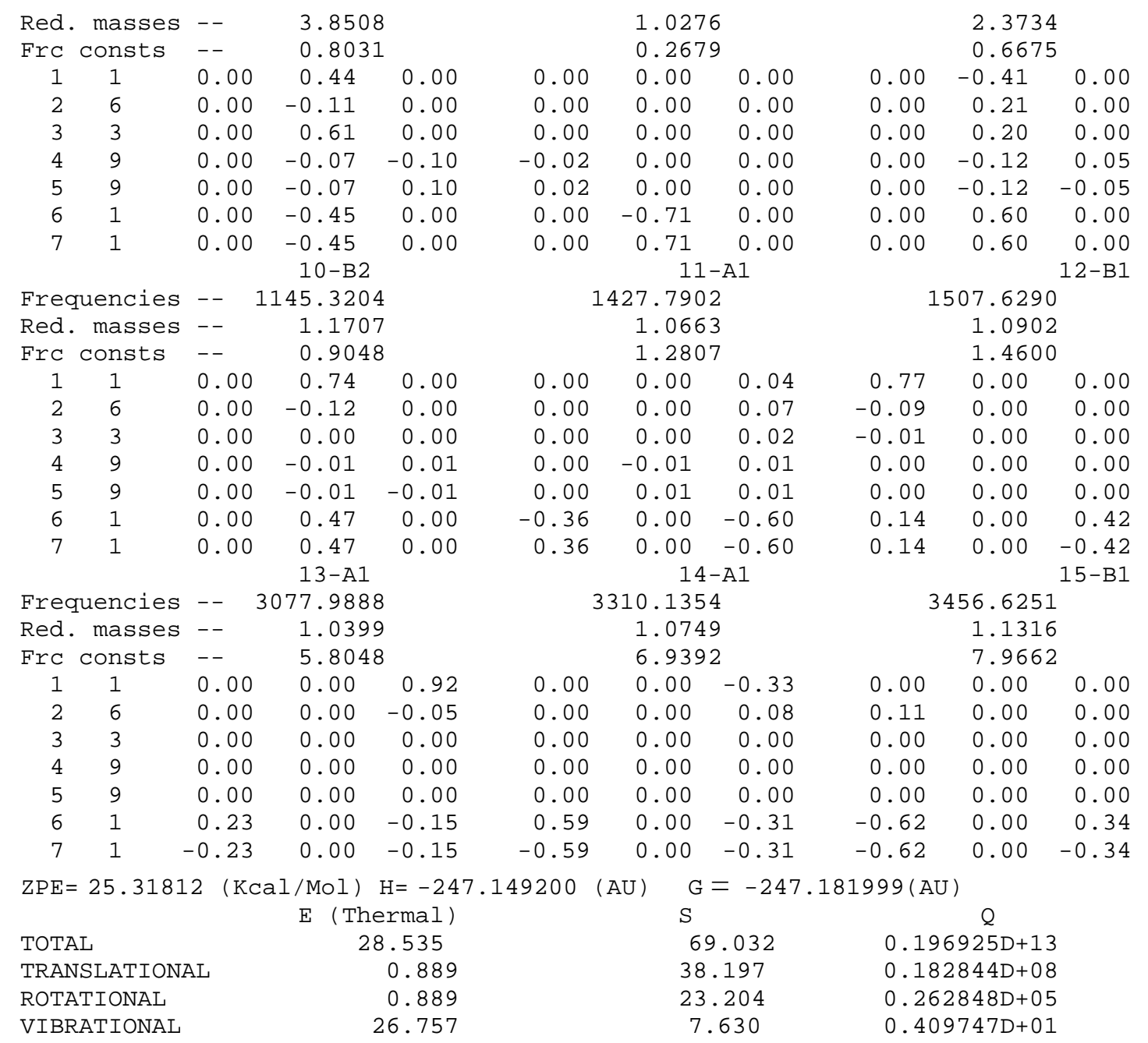

\begin{tabular}{|c|c|c|c|c|c|c|c|c|c|}
\hline TS-inv-ion & -pai & & D & $\odot$. & $\odot$. & -2.2 & & & \\
\hline LiF-CD3 - F & & & C & $\odot$. & $\odot$. & -1.1 & & & \\
\hline & & & Li & $\odot$. & $\odot$. & 1.2 & & & \\
\hline & & & $\mathrm{F}$ & 0. & 1.52812 & 0.3 & & & \\
\hline & & & $\mathrm{F}$ & $\odot$. & -1.52812 & 0.3 & & & \\
\hline & & & D & -0.95508 & $\odot$ & -0.6 & & & \\
\hline & & & D & $\odot .95508$ & $\odot$. & -0.6 & & & \\
\hline & & 1-B2 & & & $2-\mathrm{A} 1$ & & & 3-B1 & \\
\hline Frequencies & -- & -524.3133 & & & 182.1163 & & & 235.061 & \\
\hline Red. masses & - & 3.3890 & & & 7.8596 & & & 7.926 & \\
\hline Frc consts & -- & 0.5489 & & & 0.1536 & & & $0.25 \varepsilon$ & \\
\hline 11 & $\odot .0 \odot$ & 0.78 & 0.00 & 0.00 & 0.00 & 0.48 & 0.26 & $\odot .00$ & $\odot .00$ \\
\hline 2 & 0.00 & 0.25 & 0.00 & 0.00 & 0.00 & 0.47 & 0.17 & $\odot .0 \odot$ & 0.00 \\
\hline 3 & $\odot . \odot \odot$ & $-\odot .08$ & 0.00 & $\odot .0 \odot$ & $\odot .0 \odot$ & -0.29 & 0.85 & $\odot . \odot \odot$ & $\odot . \odot \odot$ \\
\hline 4 & $\odot . \odot \odot$ & $-\odot .06$ & 0.13 & $\odot . \odot \odot$ & -0.26 & -0.16 & $-\odot .24$ & $\odot . \odot \odot$ & $\odot .0 \odot$ \\
\hline 5 & 0.00 & -0.06 & -0.13 & $\odot . \odot \odot$ & 0.26 & -0.16 & -0.24 & 0.00 & $\odot .0 \odot$ \\
\hline 6 & $\odot . \odot \odot$ & -0.38 & $\odot .0 \odot$ & -0.05 & $\odot . \odot \odot$ & 0.37 & 0.17 & $\odot .0 \odot$ & -0.01 \\
\hline 7 & $\odot .0 \odot$ & -0.38 & $\odot .0 \odot$ & 0.05 & 0.00 & 0.37 & 0.17 & 0.00 & 0.01 \\
\hline & & 4-A1 & & & $5-B 1$ & & & & $-A 2$ \\
\hline Frequencies & -- & 339.4361 & & & 401.7058 & & & 475.257 & \\
\hline Red. masses & -- & 10.8561 & & & 2.2392 & & & $2.08 \varepsilon$ & \\
\hline Frc consts & - & 0.7370 & & & 0.2129 & & & 0.277 & \\
\hline 11 & $\odot .0 \odot$ & 0.00 & 0.37 & 0.50 & 0.00 & $\odot .0 \odot$ & $\odot .00$ & 0.00 & 0.00 \\
\hline 2 & 0.00 & 0.00 & 0.38 & -0.12 & $\odot .00$ & 0.00 & $\odot .0 \odot$ & $\odot .0 \odot$ & 0.00 \\
\hline 3 & $\odot .0 \odot$ & $\odot . \odot \odot$ & 0.47 & 0.09 & $\odot .0 \odot$ & $\odot .0 \odot$ & $\odot .0 \odot$ & $\odot . \odot \odot$ & $\odot .0 \odot$ \\
\hline
\end{tabular}




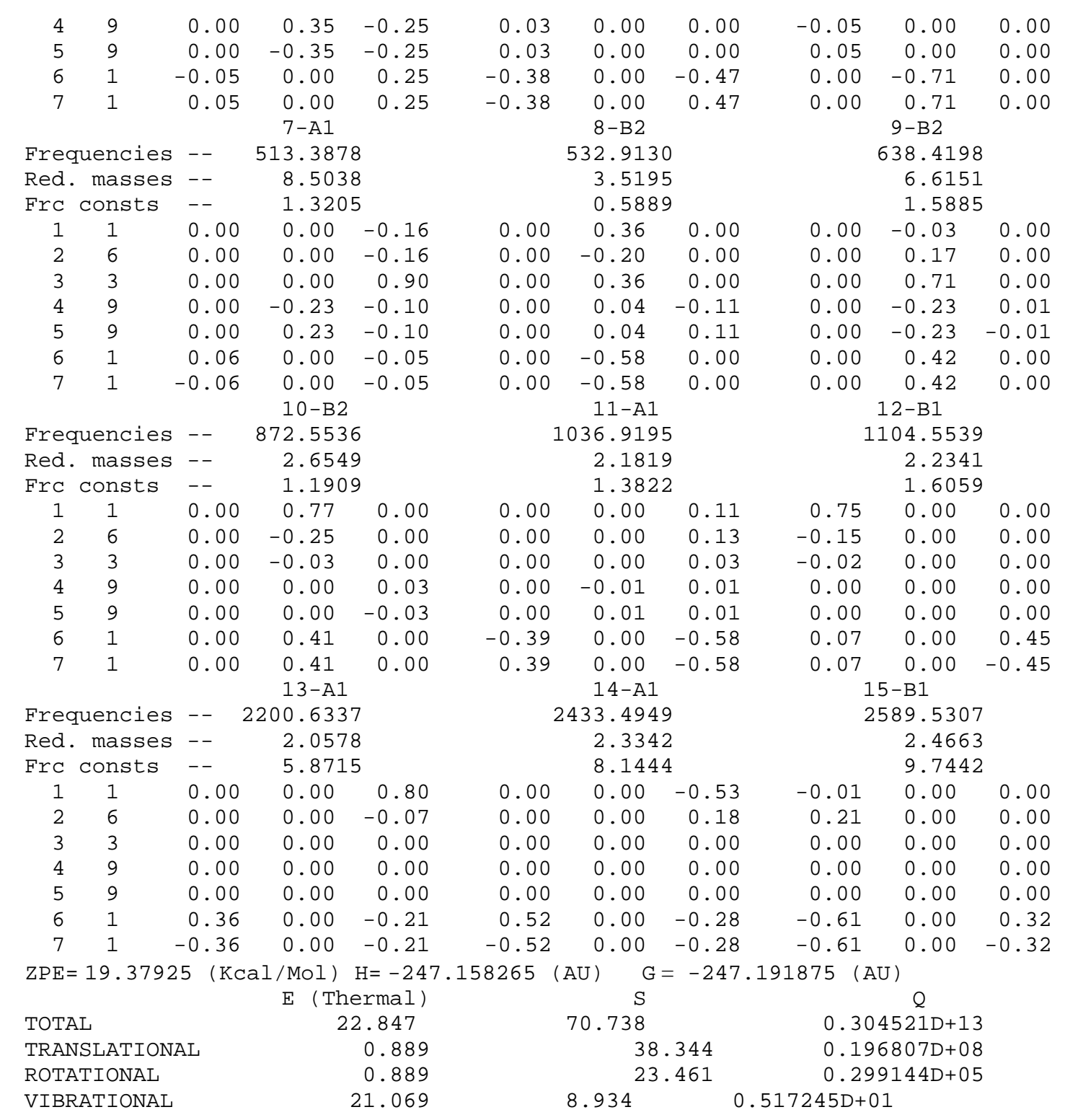

TS-ret-ion-pair $H, 0,-0.7004271061,0 .,-1.5308043098$

\section{LiF-CH3-F}

Cs
C, $\odot, \odot .3220268115, \odot .,-1.1904159936$

$\mathrm{Li}, \Theta,-\odot .3910242316, \odot ., 1.4586192017$

$\mathrm{F}, \odot,-\odot .1002410389,1.2712568696,0.3829261186$

$\mathrm{F}, 0,-0.1002410389,-1.2712568696,0.3829261186$

$\mathrm{H}, 0,0.8728388155,-\odot .9198063959,-1.2976137343$

$\mathrm{H}, \odot, \odot .8728388155, \odot .9198063959,-1.2976137343$

$$
1-A^{\prime \prime}
$$

Frequencies - - -545.9168

Red. masses - - $\quad 3.1118$

Frc consts - - $\quad 0.5464$

$\begin{array}{rrrrr}1 & 1 & 0.0 \odot & 0.0 \odot & -0.03 \\ 2 & 6 & 0.0 \odot & 0.0 \odot & 0.31 \\ 3 & 3 & 0.0 \odot & 0.0 \odot & -0.07 \\ 4 & 9 & 0.02 & 0.14 & -0.10 \\ 5 & 9 & -0.02 & -0.14 & -0.10 \\ 6 & 1 & 0.24 & -0.52 & 0.30 \\ 7 & 1 & -0.24 & 0.52 & 0.30 \\ & & & 4-A^{\prime} & \end{array}$

Frequencies - - 255.7215

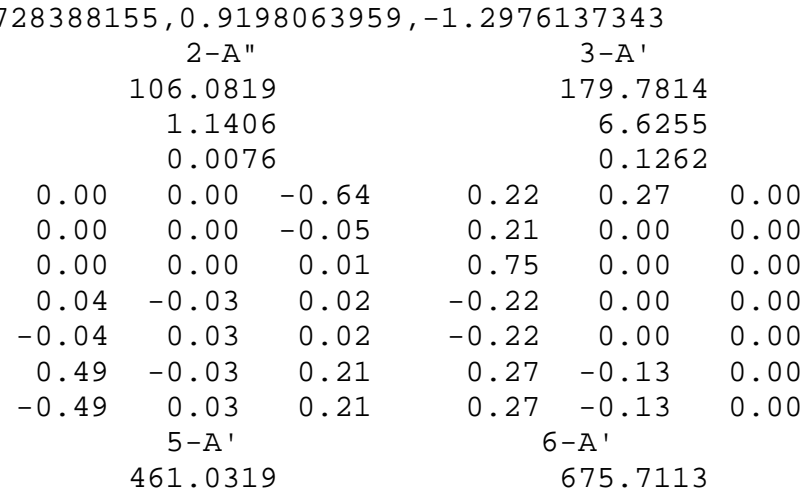

-S 9- 


\begin{tabular}{|c|c|c|c|c|c|c|c|c|c|c|}
\hline Red. & masses & -- & \multicolumn{2}{|c|}{14.4341} & \multicolumn{3}{|c|}{6.8960} & \multicolumn{3}{|c|}{8.5334} \\
\hline Frc & consts & -- & \multicolumn{2}{|c|}{0.5561} & \multicolumn{3}{|c|}{0.8636} & \multicolumn{3}{|c|}{2.2956} \\
\hline Atom & AN & $x$ & $\mathrm{Y}$ & Z & X & Y & Z & X & Y & Z \\
\hline 1 & 1 & -0.02 & -0.11 & $\odot .00$ & -0.04 & 0.61 & 0.00 & -0.01 & 0.12 & 0.00 \\
\hline 2 & 6 & -0.01 & 0.09 & $\odot .00$ & -0.03 & 0.63 & $\odot .0 \odot$ & -0.01 & 0.03 & $\odot .0 \odot$ \\
\hline 3 & 3 & -0.01 & 0.53 & $\odot .00$ & $\odot . \odot \odot$ & -0.25 & $\odot .0 \odot$ & $\odot .0 \odot$ & 0.91 & 0.00 \\
\hline 4 & 9 & $\odot . \odot \odot$ & -0.11 & 0.56 & 0.01 & -0.18 & -0.02 & $\odot .00$ & -0.18 & -0.20 \\
\hline 5 & 9 & $\odot .0 \odot$ & -0.11 & -0.56 & 0.01 & -0.18 & $\odot .02$ & $\odot .00$ & -0.18 & 0.20 \\
\hline 6 & 1 & 0.01 & -0.16 & -0.05 & 0.03 & 0.21 & $-\odot .08$ & $\odot .02$ & -0.08 & -0.01 \\
\hline 7 & 1 & 0.01 & $\begin{array}{r}-0.16 \\
7-A^{\prime \prime}\end{array}$ & 0.05 & 0.03 & $\begin{array}{r}0.21 \\
8-A^{\prime}\end{array}$ & $\odot .08$ & $\odot .02$ & $\begin{array}{l}-\odot .08 \\
9-A^{\prime \prime}\end{array}$ & $\odot .01$ \\
\hline \multicolumn{2}{|c|}{ Frequencies } & -- & \multicolumn{2}{|c|}{700.5969} & \multicolumn{3}{|c|}{814.0491} & \multicolumn{3}{|c|}{878.3419} \\
\hline Red. & masses & -- & \multicolumn{2}{|c|}{6.5599} & \multicolumn{3}{|c|}{1.1195} & \multicolumn{3}{|c|}{ 1. 2110} \\
\hline Frc & consts & -- & \multicolumn{2}{|c|}{1.8971} & \multicolumn{3}{|c|}{0.4371} & \multicolumn{3}{|c|}{0.5504} \\
\hline 1 & 1 & $\odot .0 \odot$ & $\odot . \odot \odot$ & 0.05 & -0.05 & 0.83 & $\odot .0 \odot$ & $\odot .0 \odot$ & $\odot .00$ & 0.02 \\
\hline 2 & 6 & $\odot .0 \odot$ & $\odot .00$ & $\odot .08$ & -0.08 & -0.04 & $\odot .00$ & $\odot .00$ & $\odot .00$ & 0.03 \\
\hline 3 & 3 & $\odot .0 \odot$ & $0.0 \odot$ & 0.82 & 0.00 & -0.01 & 0.00 & $\odot .0 \odot$ & $\odot .0 \odot$ & -0.07 \\
\hline 4 & 9 & 0.00 & -0.07 & -0.19 & 0.02 & 0.01 & 0.00 & 0.01 & 0.07 & -0.01 \\
\hline 5 & 9 & 0.00 & 0.07 & -0.19 & 0.02 & 0.01 & 0.00 & -0.01 & -0.07 & -0.01 \\
\hline 6 & 1 & 0.05 & 0.29 & 0.18 & 0.10 & -0.37 & $\odot .02$ & 0.05 & 0.66 & 0.22 \\
\hline 7 & 1 & $-\odot .05$ & $\begin{array}{c}-0.29 \\
10-A^{\prime}\end{array}$ & 0.18 & 0.10 & $\begin{array}{l}-0.37 \\
11-A^{\prime \prime}\end{array}$ & -0.02 & $-\odot . \odot 5$ & $\begin{array}{l}-0.66 \\
12-A^{\prime}\end{array}$ & 0.22 \\
\hline \multicolumn{2}{|c|}{ Frequencies } & -- & \multicolumn{2}{|c|}{1226.6618} & \multicolumn{3}{|c|}{1421.8520} & \multicolumn{3}{|c|}{1458.7685} \\
\hline Red. & masses & -- & \multicolumn{2}{|c|}{1.1084} & & 1.091 & & & 1.095 & \\
\hline Frc & consts & -- & 0.982 & & & 1.299 & & & 1.373 & \\
\hline 1 & 1 & -0.04 & -0.52 & $\odot .0 \odot$ & $\odot . \odot \odot$ & $\odot .00$ & $\odot .79$ & 0.08 & 0.17 & $\odot . \odot \odot$ \\
\hline 2 & 6 & -0.01 & 0.09 & $\odot .00$ & $\odot .00$ & $\odot .00$ & -0.09 & $\odot .09$ & $\odot .00$ & 0.00 \\
\hline 3 & 3 & $\odot . \odot \odot$ & -0.01 & $\odot . \odot \odot$ & 0.00 & $\odot .00$ & $\odot . \odot \odot$ & $\odot .0 \odot$ & $\odot .0 \odot$ & 0.00 \\
\hline 4 & 9 & 0.00 & 0.02 & -0.01 & 0.00 & $\odot .00$ & $\odot .00$ & 0.00 & $\odot .00$ & 0.00 \\
\hline 5 & 9 & $\odot .0 \odot$ & $\odot .02$ & 0.01 & 0.00 & 0.00 & $\odot .0 \odot$ & $\odot .00$ & 0.00 & $\odot .0 \odot$ \\
\hline 6 & 1 & 0.09 & -0.59 & -0.11 & 0.40 & $-\odot .07$ & 0.13 & $-\odot .57$ & -0.11 & -0.37 \\
\hline 7 & 1 & 0.09 & $\begin{array}{r}-0.59 \\
13-A^{\prime}\end{array}$ & 0.11 & -0.40 & $\begin{array}{r}0.07 \\
14\end{array}$ & $\begin{aligned} & 0.13 \\
-A^{\prime \prime} & \end{aligned}$ & -0.57 & -0.11 & $\begin{array}{c}0.37 \\
15-A^{\prime}\end{array}$ \\
\hline Freq & |uencies & -- & 3140.615 & & & 3301.557 & & & 317.806 & \\
\hline Red. & masses & -- & 1.014 & & & 1.116 & & & 1.11 & \\
\hline Frc & consts & -- & 5.893 & & & 7.171 & & & 7.25 & \\
\hline 1 & 1 & 0.55 & -0.03 & $\odot .0 \odot$ & $\odot .0 \odot$ & 0.00 & 0.01 & 0.84 & -0.03 & $\odot .0 \odot$ \\
\hline 2 & 6 & 0.00 & 0.02 & $\odot .00$ & 0.00 & $\odot .00$ & 0.10 & -0.10 & -0.01 & 0.00 \\
\hline 3 & 3 & $\odot .0 \odot$ & 0.00 & 0.00 & 0.00 & $\odot .00$ & $\odot .0 \odot$ & $\odot .0 \odot$ & $\odot .0 \odot$ & 0.00 \\
\hline 4 & 9 & $\odot .0 \odot$ & $\odot .00$ & $\odot .00$ & 0.00 & $\odot .00$ & 0.00 & $\odot .00$ & 0.00 & 0.00 \\
\hline 5 & 9 & $\odot .0 \odot$ & $\odot .00$ & $\odot .0 \odot$ & 0.00 & $\odot .0 \odot$ & $\odot .0 \odot$ & 0.00 & 0.00 & 0.00 \\
\hline 6 & 1 & -0.27 & -0.12 & 0.51 & 0.33 & 0.15 & -0.60 & 0.17 & 0.07 & -0.33 \\
\hline 7 & 1 & -0.27 & -0.12 & -0.51 & -0.33 & -0.15 & -0.60 & 0.17 & $\odot . \odot 7$ & 0.33 \\
\hline $\mathrm{ZPE}=2$ & 25.64449 & $9 \quad(\mathrm{Kca}$ & 1/Mol) & $\mathrm{H}=-247$. & 3250 & U) $\quad G$ & $=-247$ & 7274 & & \\
\hline & & & E (Th & ermal) & & S & & & Q & \\
\hline TOTA & & & & 8.956 & & 1.610 & & $4016 \mathrm{D}+$ & & \\
\hline TRAN & SLATION & & & 0.889 & & 38 & 197 & 0.18 & $2844 D+C$ & \\
\hline ROTA & TIONAL & & & 0.889 & & 24. & 232 & 0.4 & $\odot 801 \mathrm{D}+\mathrm{C}$ & \\
\hline VIBR & ATIONAL & & & 27.179 & & 9.181 & & $1826 \mathrm{D}+$ & & \\
\hline
\end{tabular}

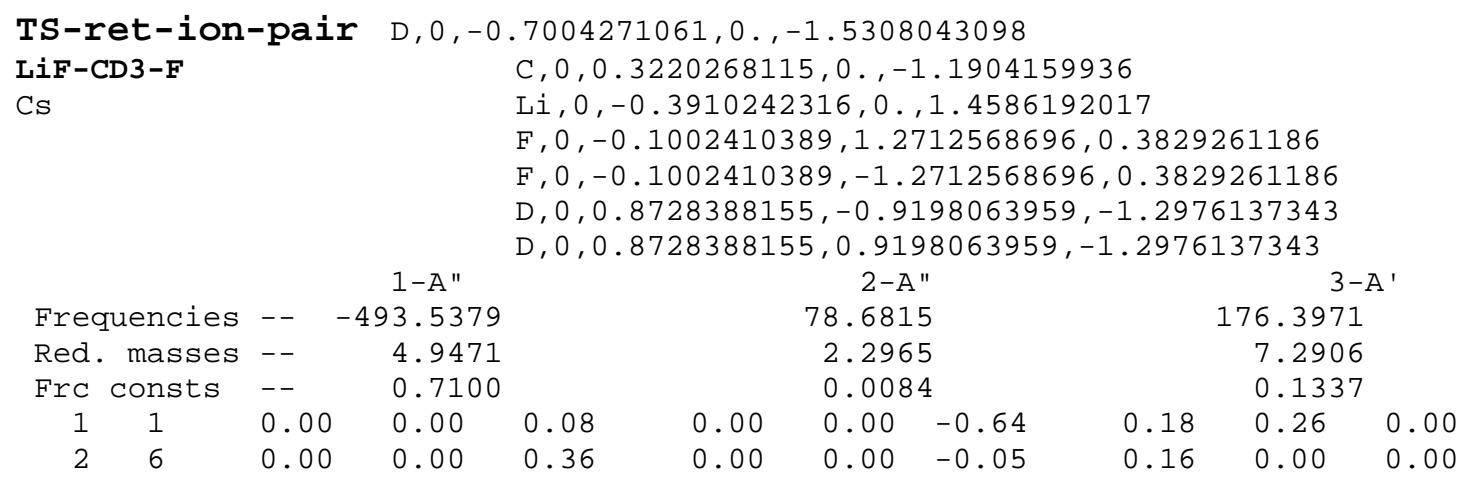




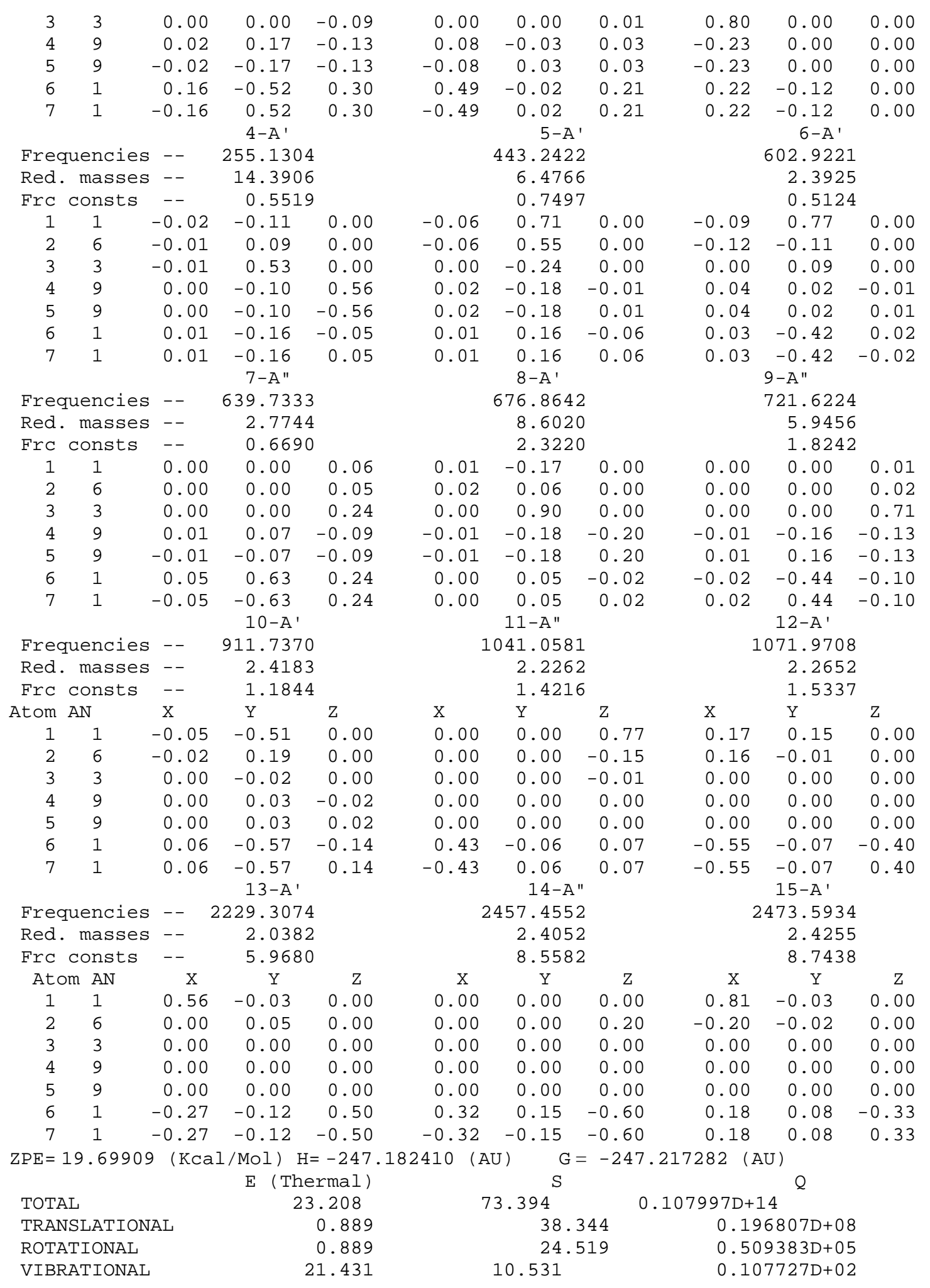

